\title{
Die Bootfahrt ins Jenseits
}

\author{
Von M. Ebert
}

Auf dem von Paribeni veröffentlichten bekannten Tonsarkophag ron Hagia Triada ${ }^{1}$ ) ist auf der einen Seite eine Szene vor einem Grabe dargestellt (Abb. 1). Der Tote, neben dem ein Baum und ein Podest mit treppenartigem Aufgang angedeutet sind, empfängt vor seinem Grabeshause die ihm gebührenden Opfer. Männer in einem eigenartigen, viel besprochenen weißen Fell um den Unterleib und die Beine überbringen ihm

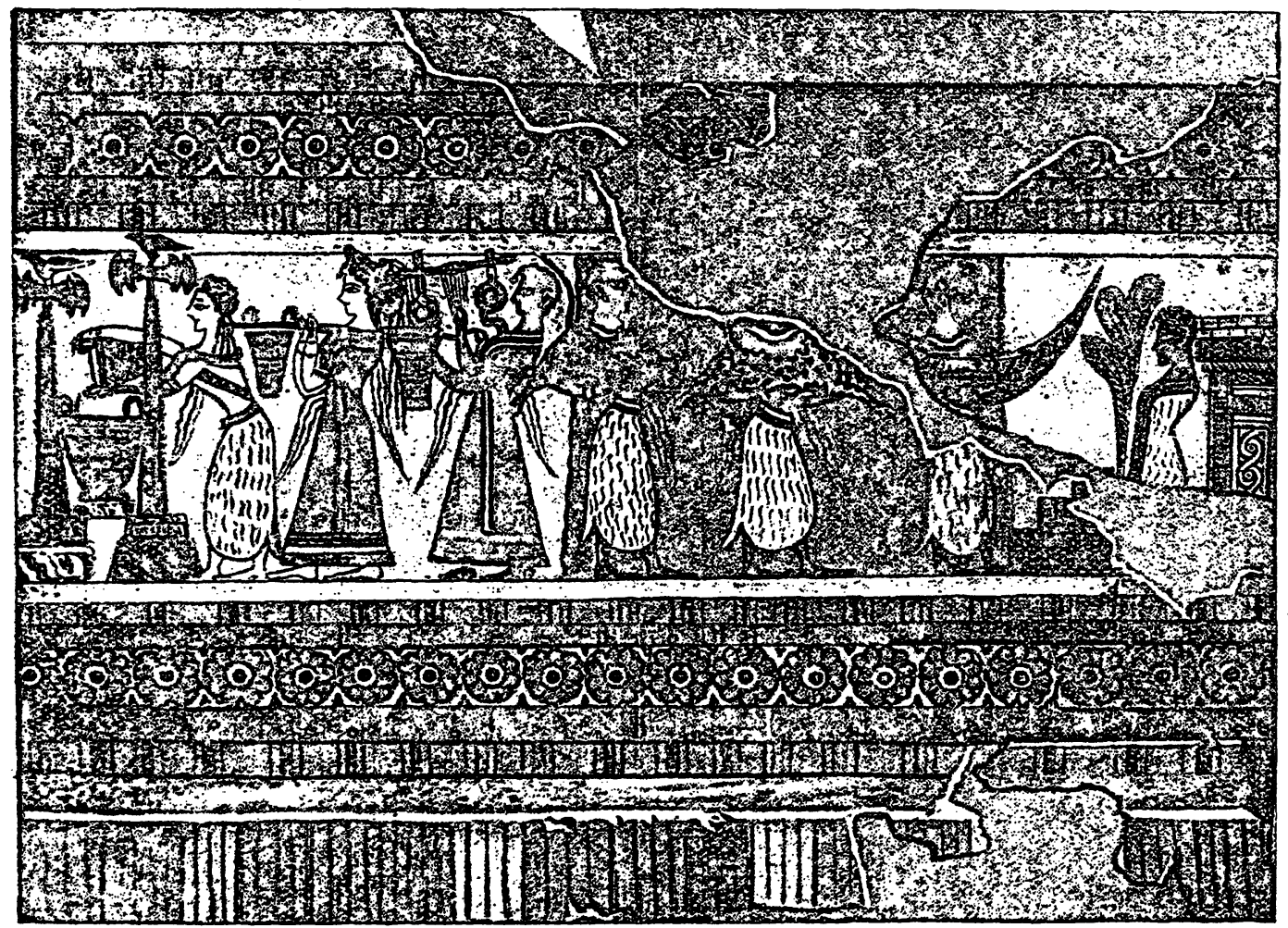

Abb. 1.

Rinder und ein Schiff. Was hat dieses Schifi zu bedeuten? Es ist entweder ein kleines Fahrzeug, das auch ein einzelner Mann tragen konnte, oder ein Abbild von einem solchen. Jedenfalls, ob wirkliches Boot oder nicht, der Tote empfängt ein Fahrzeug, mit dem er über ein Wasser fahren kann.

Fahrzeuge, Schiffe, Wagen, Schlitten spielen im Totenkultus des Altertums eine beträchtliche Rolle und treten demgemä $B$ auch zahlreich in den (jräberfunden auf. Viele von diesen Beförderungsmitteln haben eine rein praktische Bedeutung. Die Friedhöfe lagen häufig in einiger Entfernung von den Wolnstätten, die den letzten Weg des Abgeschiedenen zu einer kleinen, manchmal auch $2 u$ einer größeren, ziemlich umständlichen Reisc

1) Monumenti dei Lincei XIX (1908) S. 5 ff. 


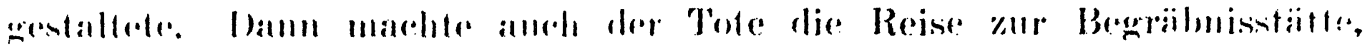
mil allem was ihn begleitete, anf rincm Wagen oder, wio \%. ls. inn Niltale. we die Verkehesverhailtnisse das mahelegten, anf einer barke.

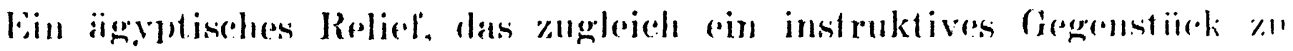

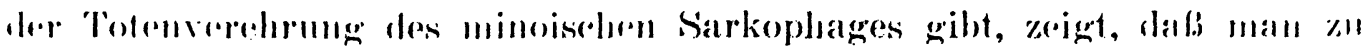

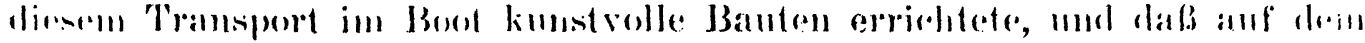

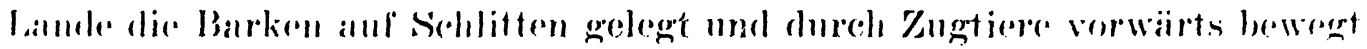
wirrlen. :-)

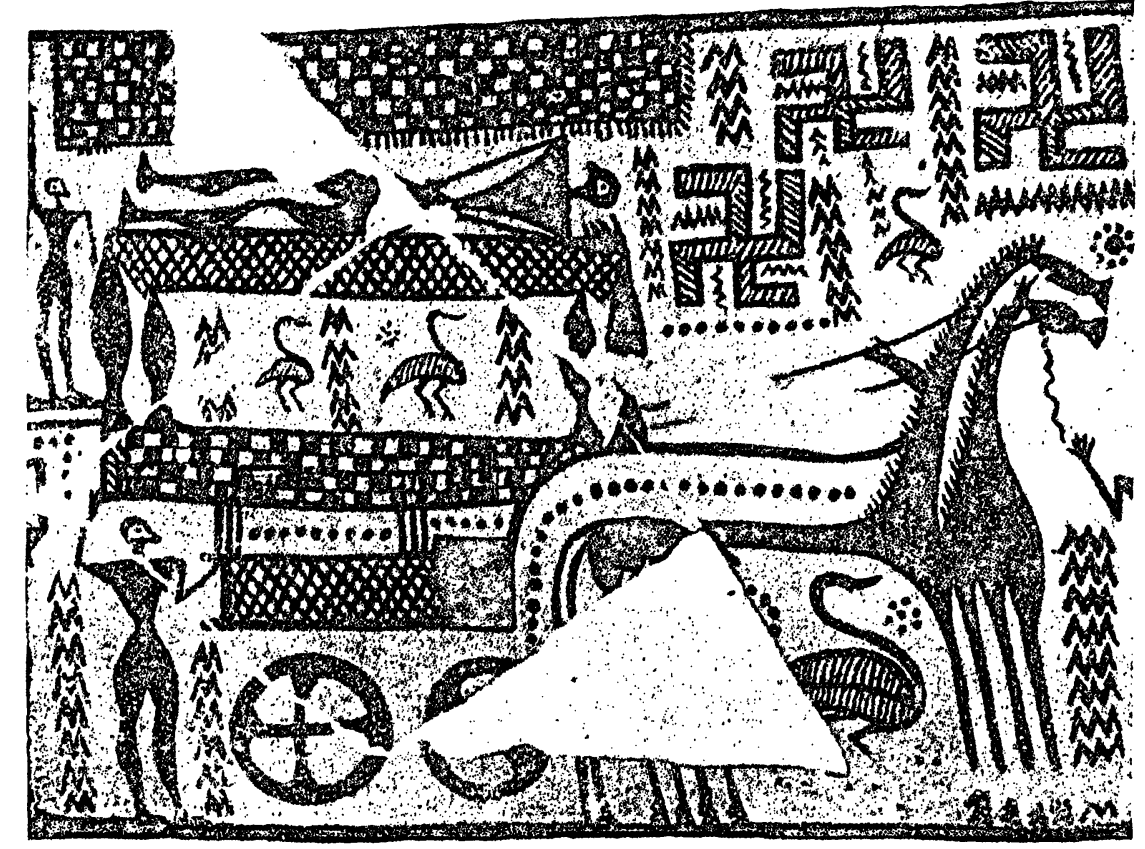

Abb. 2.

ben 'lransport zu Wagen zeigt eine 1)ipylonvase des Athener Nationalmusemus (Abb. 2).3) Auf dem Wagen - oder vielmehr nicht direkt auf ihm, sondern anf einem, hier schachbrettartig gemusterten Podium, das in der Länge und Breite über den Kasten hinausragt nud von einem Bowleiter gestiityt wirl, steht die Kline des 'Toten. Sie hat geolroehselto Brome und ein lagere, das anf diagonal gekrenzten Gurten ruht. Wir werten ums das und die Decke, die nach der primitiven Ausdrucksweise des gonmotrischen Vasenkïnstlers wohl über den 'Toten gebreitet zu druken ist. nolir präichtig vorstellen müissen.

In skythischen Gräbern des 6.-3. Jahrhumblerts sind oine Anzahl ron Wagen wefunclen, die aujschließlich als Teichenwagen dienten mol fiir diesen Zweck gebaut waren. ${ }^{4}$ ) Zu den aitesten gehören die Reste von

2) A. Eiman, Aegypten 11336 .

3) Mon. d. Inst. IX. 39, 1; Collignon-Couve, Calalogue des vases d'Athènes No. 214; liurt Müller, I)er Leichenwagen Alexander des Großen 1005, S. $11 \mathrm{ff}$.

4) Rostowzew, Die antike dekorative Wandmalerei in SüdruBland 'rliss.' 1914 $147 \mathrm{ff} ., 511$. 
solchen Zelt- oder Baldachinwagen aus einem Kurgan bei der Stanitza Ol (Bezirk Maikop) ${ }^{5}$ ) und zwei Kurganen ebenfalls im Kubangebiet ${ }^{6}$ ) mit apotropäischen Schmuck an den Enden der Wagenachsen. Wahrscheinlich gleichzeitig, aus dem *6. Jahrhundert; sind die Reste eines Katafalkwagens von Kelermes ${ }^{7}$ ) mit sehr interessanten Stangenaufsätzen archaischen Types. In den Funden des 5. und 4. Jahrhunderts fehlen Wagen bislang. Eine jüngere Reihe (3. Jahrhundert) stammt aus den Kurganen von Alexandropol, ${ }^{8}$ ) Krasnokutsk (2 Wagen) ${ }^{9}$ ), von Slonowskaja Blisnitza (mit einer späten rotfigurigen Kylix), ${ }^{10}$ ) Tschertomlyk, ${ }^{11}$ ) aus anderen Plätzen des Dnjeprgebietes und aus Ungarn. ${ }^{12}$ ) Leider ist bisher kein vollständiges Exeniplar eines solchen Wágens gefunden und ein Rekonstruktionsversuch noch nicht unternommen. Es scheint; daß das Obergestell ron langen Stangen gebildet: wurdè, die oben in eisernén oder bronzenén Aufsätzen endeten. Das waren bei den archaischen Wagen meist durchbrochen gearbeitete ei- oder kugelförmige Stücke, innen mit kleinen Kugeln gefüllt. Bei der Bewegüung erklangen diese Glöckchen; ịndem sie gegen die Wände der Kngel schlugen und vereinigten : ilhr. Geläut mit dem von Glöckchen, die an die Stangen und andere Teile des Wagens gèhängt waren. Die Aufsätze der jüngeren frühhellenistischen Leichen wagen endeten in der Figur einer Gottheit oder eines heiligen Tieres.. Das Gerüst war mit Teppichen bedeckt, von denen Blechschildchen zusammen mit Wagenresten in einigen Gräbern gefunden sind. Diese Baldachinwagen der skythiṣchen Könige gehen unzweifelhaft auf dieselben Traditionen zurück wie der von Diodor geschilderte:Leichenwagen, auf dem der Körper. Alexanders dès Großen von Rabylon nach Memplịs überführt wurde, ${ }^{13}$ ) nämilich auf den Reisewagen der iranischen Nomaden. Modelle davor ' sind in grièchischen Gräbern bei Kertsch gefunden.14) Besonders in Betracht kommen Wagenformen mit Oberteil in Gestalt einer Kamera oder-einer zylindrischen Wölbung (Herodot, I 199), die an die Begräbniswagen auf dem Friese des Pleureusensarkophages von Sidon erinnern. Möglicherweise sind diese Kertscher Terrakottawägelchen, zu deren auch ein Exemplar aus einem Ockergrab von Ol $\left.{ }^{15}\right)$ kommt, nicht nur Kinderspielzeug, sondern wirkliche Bestattungsrequisite, Symbole des Reisewagens, den der Tote im Jenseits benutzen sollte.

Ersprünglich ein von den iranischen Wandervölkern geschaffener und benutzter Typus wurde der Katafalkwagen, wie Rostowzew in seinem in-

5) Pharmakowski im Arch. Anzeiger 1910 Sp. 196 ff:

6) Atschot 1908 S. 118.".

7) Arch. Anzeiger $1905 \mathrm{Sp} .57 \mathrm{fi}$.

8) Altertümer des Herodot. Skythiens (russ.〉 I. 21. - 9) ibid. II 44. - 10) ibid. II 65. - 11) ibid. II 79.

12) G. Nagy, A Skythak 1909 S. 48 ff.

13) Kurt Müller, Der Leichenwagen Alexandèrs des Grofen 1905; Bulle, Arch. Jahrbuch XXI (1906) S. 52 ff.; Thiersch ibid. XXV (1910) S. $55 \mathrm{ff}$; v. Wilamowitz ibid. 1905 S. 104.

14) Bienkowski, Wiener Studien 1.902 S. 394 fi.; v. Stern, Bobrinskoi-Festschrift 1911 S. 26; Rostowzew a. a. 0. I. 49 Abb. 10, 11.

15) Arch. Anzeiger 1910 Sp. 196; Iswestja der archäol. Kommission 35 S. 3. 
haltreichen Buch dargetan hat, in Persien unter altionischem Einfluf im 6. Jahrhundert zu einein von Lebenden und Toten benutzten Paradewagen umgestaltet. In der Zeit des frühen Hellenismus hat ein Künstler bei einem großen Anlaß, dem Bestattungszuge Alexanders, die alte Form benutzend, ein neues Vorbild geschaffen, das dann vielfach nachgeahmt wurde.

In Südrußland sind die Baldachinwagen nach dem 3. Jahrhundert v. Chr. nicht melır üblich. Wahrscheinlich wurden sie durch die auch früher schon verwendeten tragbaren Katafalke abgelöst. ${ }^{16}$ )

Es sind also diese Wagen, auf denen man, wie Herodot (IV 71) erzählt, die Leichname der Könige, nachdem man sie einbalsamiert hatte, bei allen Stämmen herumfuhr. Dann erst werden sie im Lande Gerrhos, bei den Stromschnellen des Dnjepr-Borysthenes unter blutigen Totenopfern bestattet. „Die übrigen Skythen aber, wenn sie gestorben sind, fahren die nächsten Verwandten auf einem Wagen umher zu ihren Freunden. Die nehmen sie alle auf und geben dem Leichengefolge einen Schmaus und setzen der Leiche von allem ebenso vor wie den übrigen.". Man bestattete die Toten nicht gleich, sondern läßt sie noch eine Weile unter den Lebenden weilen, je vornehmer der Tote ist; um so länger. Die Leiche des dänischen Königs Frotho wird noch drei Jahre aufbewahrt, auf einem mit Ochsen bespannten Wagen herumgefahren, wie Saxo berichtet ,ut jam non funebri lecto, sed regali vehiculo gestari videretur" und dann erst beigesetzt. ${ }^{1 T}$ ) Wulfstan erzählt von den alten Preußen, daß sie die Toten einen Monat oder zwei, die Fürstén manchmal ein halbes Jahr unverbrannt in ihren Häusern liegen ließen. Und alle diese Zeit ehrte man sie durch Schmaus und Trunk. ${ }^{18}$ )

Bei den wenigstens $z$. T. noch nieht seßhaften und zerstreut wohnenden Skythen holt also der Tote auf seinem Wagen sich die ihm zustehenden Ehren selbst ein. Im übrigen liegt dieselbe Anschauung zugrunde. Schion für die vorhin angeführte Dipylonvase hat Studniczka die Vermutung ausgesprochen, daß der Wagen der Streitwagen der Toten sei, dem man nur für den funeralen Zweck herrichtete, indem man die Geländer um den Wagenkasten herum abnahm. Die Unbeholfenheit der Darstellung und der Mangel an Denkmälern ăus der geometrischen Stufe erlaubt nur, das als eine sehr wallrscheinliche Vermutung anzusehen. Wagen dieser Kategorie haben uns besonders die thrakischen, ${ }^{19}$ ) italischen und keltischen Nekropolen geschenkt. Einer von ihnen, der Bronzewagen von Monteleone di Spoleto bei

16) Kurgan von Maikop: Pharmakowski in Materialien zur Archäologie Rußlands 34 (1914); - Große Blisnitza: Rostowzew I 12.

17) Uhland, Schriften zur Geschichte der Dichtung und Sage VII S. 114; Sartori in der Eduard Hahn-Festschrift:1917, S. 241.

18) Scriptores rerum prussicarum vol. I 730. Ähnlich bei den Esten Schivenck, Mythologie der Slaven S. 428; Ein "survival" dieser Sitte am französischen Königshofe: Tylor (deutsche Ưbersetzung von Spengler und Poske) II 34.

19) Seure, Bulletin de corr. hellén. XXV (1901) S. 181 ff.; B. Filow, Arch. Anzeiger XXV (1910) S. 401. 
Norcia, der Besitz eines italischen Fürsten des 6. Jahrhunderts, gehört zu len. vortrefflichsten und vollständigsten archaischen Netallarbeiten, die erhalten sind (Abb. 3). ${ }^{20}$ ) Er stellt zugleich eines der Bindeglieder clar zwischen den Streitwagen der Kleinasiatischen Griechen und den Wagen der

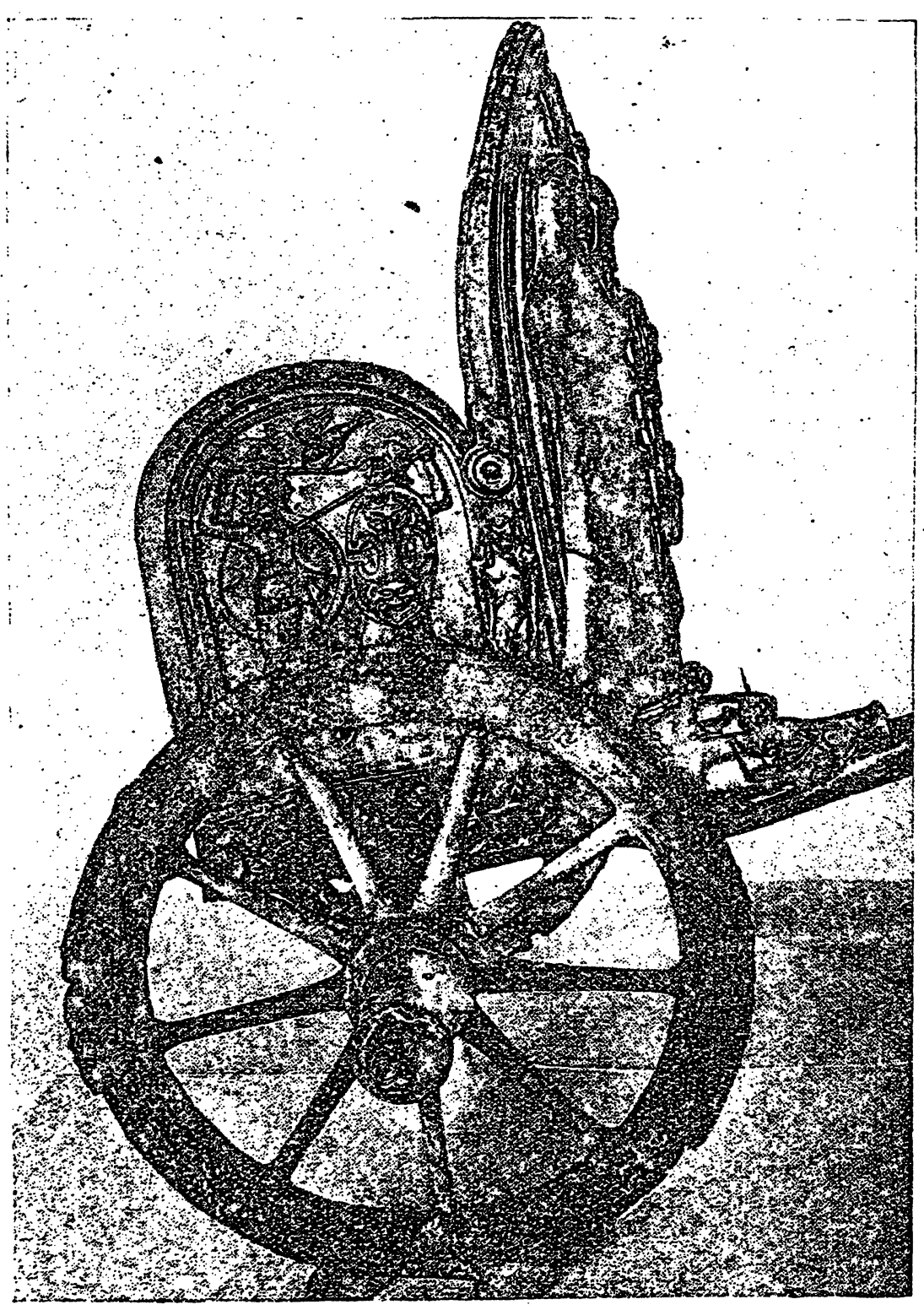

Abb. 3

keltischen Fürstengräber der La Tènezeit. ${ }^{21}$ ) Vielleicht gehört aus dem germamischen Norden das Brandgrab ron Kraghede bei Hjörring (Däne-

20) Furtẉängler in Brunn-Bruckmanns Denkmäler Griech. und Röm. Skulptur Taf. 586 , $58 \mathrm{t}=\mathrm{Kl}$. Schriften 11 314. ff.

21). J. Déchelett, Manuel II 3 S. 1180. 
mark) dazu, wo man auf dem Scheiterhaufen den Wagen des Toten mit zwei vorgespanuten Rossen verbraunte. ${ }^{22}$ )

Zwischen solchen Fahrzeugen, wie den Totenbarken der Ägypter, den Leichenwagen der geometrischen Zeit Griechenlands und skythischer Kurgane auf der: einen Seite und diesen Streitwagen auf der andern besteht, die dem Ritus zugrunde liegende Idee angesehen, ein Unterschied. Jene gehören zum Requisit der Bestattungszeremonie, sie sind die Transportmittel, um den Toten rom Flause zum Grabe zu bringen, diese - mag

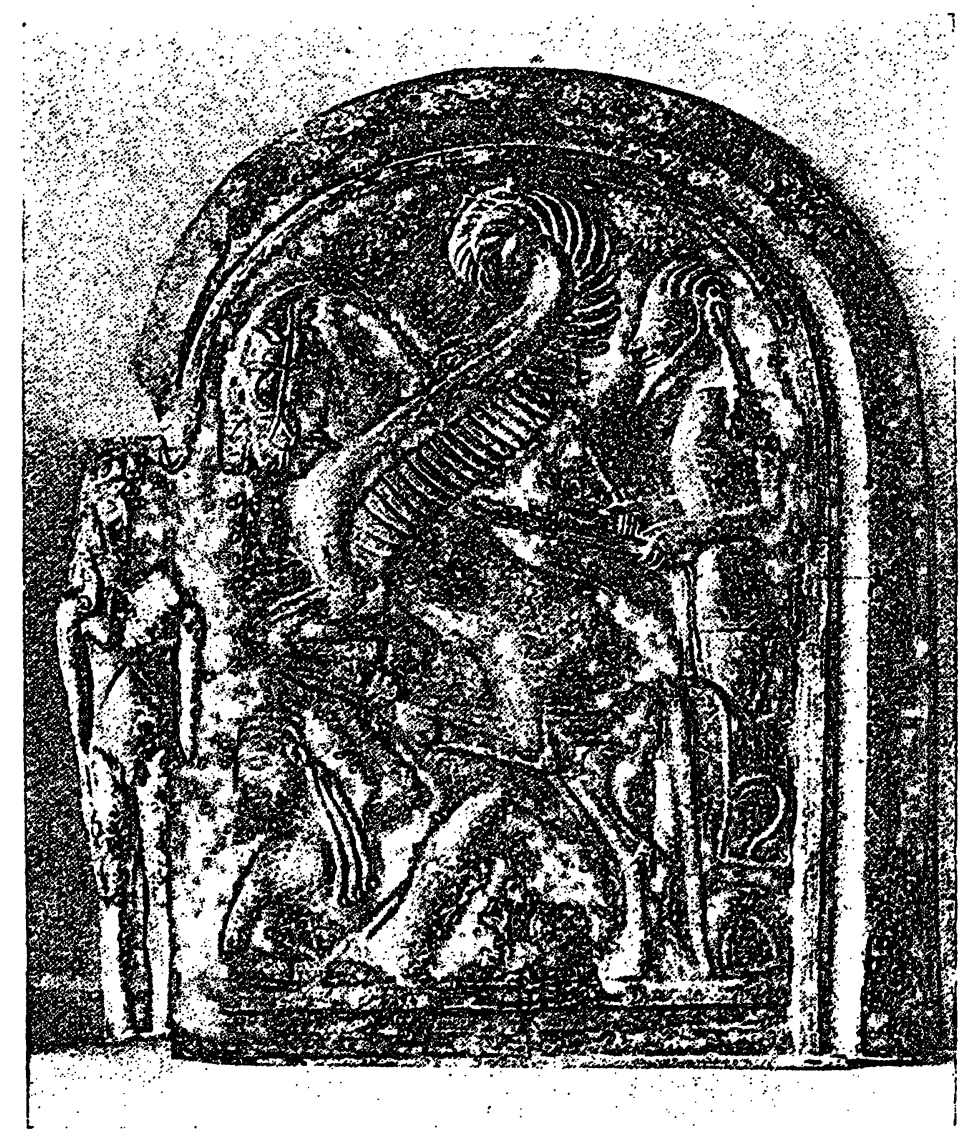

Abb. 4 .

man sie auch zum selben Zwecke verwendet haben - bilden glcichzeitio einen Teil der Grabaussteier, wie die Waffen, der Schmuck und die Nah. rung, die man dem Verstorbenen mitgibt. Er brancht sie im Jenseits. uin dort wie im Diesseits seine Feinde bekämpfen zu können..

Die Darstellung auf der rechten Nebenseite des Wagens von MIonteleome führt uns gleich einen Schritt weiter (Ab). 4). Der Held erhebt sich anf einem Wagen, der von geflügelten Rossen gezogen wird, hinweg; ron der

22) Führer durch das dänische Nationalmuseum 1911, No. 213, 214. - Öber weitere germanische Begräbnisse mit Wagen Mötefindt und Sartori in der Hahn-Festschrift 1917 S. 214, 244. 
Erde, die als liegende Frau im Chiton dargestellt ist, in die Lüfte. Szenen ähnlicher Art auf Bologneser Grabstelen, lassen keinen Zweifel daran, daß damit die Fahrt ins Jenseits gemeint ist. ${ }^{23}$ )

Die Fahrzeuge, die also 1. als funerale Transportmittel, 2. als Grabausstewer dienen, erscheinen hier 3. als Beförderungsmittel ins Jenseits. ${ }^{2+}$ ) Diese Rolle ist sicherlich auch der Barke zugedacht, die dem Toten auf dem Sarkophage von Hagia Triada als Grabspende dargebracht wird, wie auf zahlreichen Booten und Schifflein, die Paribeni, aus Grabfunden, von Grabreliefs oder sonst mit dem Totenkultus der Mittelmeerländer zusammenhängenden Denkmälern aufzähltt. ${ }^{25}$ )

Schon im Kreta der spätminoischen Epoche und in vielen anderen Gebieten des Mittelmeeres glaubte man also, wie in der homerischen und späteren Zeit, daß das Diesseits rom jenseitigen Lande durch ein Gewässer getrennt sei, das der Tote mit einer Barke überschreiten müsse.

Aber nicht nur dort. Man findet diese Vorstellung bei zahlreiclien Völkern des Altertunis, bèi den Bewohnern außereuropäischer Erdteile und in den Sagen, Märchen und im Aberglauben wohl bewahrt oder in Überbleibseln des mittelalterlichen und neueren Europás. ${ }^{26}$ ) Nirgends hat aler die Idee im Grabbau und Totenkultus einen so bedeutenden, teilweise monumentalen Ausdruck gefunden als in den Boots- .und Schiffsgräbern nordgermanischer Stämme in :der zweiten Hälfte des 1. nachchristlichen Jahrtausends. Es ist kein Zufall, daß die Sitte, die Toten in Barken und. Schiffen zu begraben, gerade in dem fjordreichen Norwegen und in dem von zahlreichen Flüsssen und Seèn dürchzogenen Mittelschweden besonders verbreitet war und in einer. Zeit in Aufnahme kam, wo der Handel mit den benachbarten Küstenländein stark zunahin und die unternehmungslustigen Elemente aufs Meer hinauszog. Der Kolonisation und den Eroberungszügen und Staatengründungen seit dem 8. Jahrhundert geht auch hier, wie gewöhnlich, eine. Zeit voraus, wo der Kaufmann Wege suchte, das Terrain sondierte und anknüpfte: Die Aufmerksanikeit ist erneut auf diese Schiffsgräber gelenkt worden durch einen besonders glänz̈enden Fund, der im Jahre 1903 bei Oseberg gemacht wurde. Oseberg ist ein Gehöft an der Westküste des Christianfjords im sülöstlichen Norwegen. Dicht bei dem Gehöft lag ein äußerlich nicht melır sehr ansehnlicher Hügel, der ein Schiff und in ihm eine hölzerne Grabkammer barg. In diesem Schiff ist

23) Ducati, Rend. Lincẹi 1910 S. 266 ff:; ders., Le pietre funerarie felsinee, Monum. Lincei 1911 S. $357 \mathrm{ff}$.

24) Es gehört in diese Reihe auch das Pferde als Tragtier: Malten, Arch. Jahrb. 23 (1914) S. $179 \mathrm{ff}$.

25) Paribeni, Monumenti dei Lincei XIX (1908) S. 24 ff.; Gruppe, Griechische Mythologie 1651, 1; Pagenstecher, Sumbolae litterariae in honorem Julii de Petra 1911 S. 62 ff.; Arch. Jahrb. XXV (1910) S. 182; XXX (1917) S. 101, Abb. 23; Hoernes, Urgeschichle der bildenden Kunst $1915^{2}$ S. 502 ff.; Dieterich, Mithraslithurgie ${ }^{2}$ S. 183, 235.

26) Viel Literatur dazu zusammengetragen von K. Stjerna, Essays on Beowulf 1912 S. 103 ff. Vgl. besonders Tylor, Primtive Culture II 84, I 426; Wuridt, Völkerpsychologie 11 3, 561 fi.; J. Grimm, Mythologie S. 790 fi. 
wu das Jahr 850 n. Chr. eine vornehme Frau - nach A. W. Bröggers ansprechender Vermuting die Königin Aasa, die Mutter Halvdans des Schwarzen ${ }^{27}$ ) - mit einer Dienerin, die ihr ins Grab nachfolgen wollte orler mußte, bestattet worden. Sie war umgeben von einer Aussteuer für die Fahrt ins Jenseits - es interessieren uns dabei besonders der Wagen und die Schlitten - die unter den nordischen Funden bisher nicht ihres gleichen haben. Die Konservierung und meisterliche Wiederherstellung. les ron dem inzwischen verstorbenen Prof. Gustafson untersuchten Fundes erforlerte ein volles Jahrzelint; ehe man an die wissenschaftliche Veröffentlichung gehen konnte. 'Von A. W. Brögger energisch gefördert, liegt num der erste Band dieses groß angelegten und munifizent ausgestatteten Werkes ror, der eine eingehende Würdigung der norwegischen Schiffsgräber ron H. Schetelig: Hand enthält. ${ }^{28)}$

Die Bootsfahrt ins Jenseits gehört in der germanischen Spätzeit offensichtlich zu den bei Geringen und Vornehmen allgemein verbreiteten Glaubenssätzen über das Leben nach dem Tode. An dem archäologischen Naterial läßt sicb seit dem 3. nachchristlichen Jahrhundert das Aufkeimen solcher Vorstellungen und ihre inmer stärkere Materialisierung beim Totenkult verfolgen. In der römischen Kaiserzeit wird die antike Sitte, dem Toten eine oder mehrere Münzen als Fährgeld mitzrigeben, im römischen Grenzgebiet von den Germanen vielfach aufgenommen, wie das Auftreten der Charonspfennige in germanischen Friedhöfen bei Selzen, Oberolm, Trier, Lêde (Ostflandern), Bruch a. d. Leitha und anderen Orten bezeugt. ${ }^{29}$ ) Wahrscheinlich ist das nichts weiter als äußerliche Aufnabme fremden Branches. Etwas melir bedentet es sicher, wenn man diese Sitte im Zụsammenhang mit anderen Erscheinungen und der sjäteren Entwicklung seit.dem 3. Jahrhundert in Norddeutschland und Skandinativen beobachtet. Römische Goldmänzen oder goldene Zahlringe liegen in den Bestattungsgräbern im Munde der Toten. In den Brandgräbern sind sie fast immer unverbrannt, anch da, wo das übrige Grabgut anf dem Scheiterhaufen rrar, dem Leichenbrand beigegeben. ${ }^{30}$ )

Dịese Gräber dès 3-6. Jahrhunderts geben đlen ältesten Anhaltspunkt dafür, daß män sich seit spätrömischer Zeit in Germanien über den Weg ins Jenseits und die Art, wie die Seele iln'zurücklegt, Vorstellungen macht, die im rituellen Niederschlag beim Grabkultus den antiken gleichen. Sie brauchen keineswegs unkräftiger sein wie später, als man dem Toten ein

27) A. W. Brögger, Borrefundet og. Vestfold kongernes graver. Kristiania 1916.

28) Osebergfundet. Utgit av den Norske stat under redaktion av A. W. Brögger, Hj. Falk, Haakon Schetelig. Band I. Kristiania 1917. Mit einem deutschen oder engliśchen Auszug.

29) L. Lindenschmit, Handbuch der deutschen Altertumskunde I $133 \mathrm{ff}$; K. Wein:hold, SB Wien 30 (1859) S. 189 ff.

30) Himlingöie: Aarböger 1897 S. 220 ff.; Hove: 0. Rygh; Nordiske Oldsager Abb. 306, Text S. 15; Gloppen: H. Schetelig, Vestlandske graver fra jernalderen 1912 S. 50; Kälder: O. Almgren in Montelius-Festschrift 1903 S. 89 ff.; Kittendorf: Praeh. Z. II (1910) S. 195; Lunde: H. Schetelig a. a. O. S. $68,110$. 
wirkliches Boot mitgab. In Gegenteil, die sakrale Idee wird durch ihre Ausprägung im Kult, in der „beiligen Handlung“ geschwächt und zum Erstarren gebracht.

Aus dem 6. und 7. Jahrhundert stammen die ältesten Bootsgräber (Orlinshügel bei Upsala, Byrkje im norwegischen Westland, Björnes im Trondheimischen, die ältesten Wendelgräber). ${ }^{31}$ )

Wird der Tote bestattet, so legt man ihn in sein Boot, ist Leichenverbrennung Sitte, so kommt anch das Boot auf den Scheiterhanfen. Die geographische Verbreitung dieser frühesten Bootsgräber - wir kennen sie aus Norwegen, Schweden, Öland, Finnland und England - in beschränkter Zahl zeigt, daB die Sitte schnell nach Osten und Westen weitergerrandert ist. Znnächst scheint dieser Brauch sich auf die sozial und wirtschaftlich bevorzugten Kreise beschränkt zu haben. Ganz natürlich, denn ein Boot. Tar. immerhin ein Wertgegenstand. Die prächtigen Bootsgräber von Wendel in Jppland, in denen ron $650 \mathrm{n}$. Chr. ab die Angehörigen eines edlen Geschlechtes bestattet wurden, zeigen, wie diese Bestattungssitte in der Tradition einer fürstlichen Familie mehr als 300 Jahre lang fortgesetzt wrurde. Es hängt sicherlich mit dem zunehmenden Wohlstand in der Wikingerzeit zusammen, daß namentlich in Norwegen die Bootsbestattung. eine so riesige Verbreitung fand: Utber 550: Bootsgräber ats dieser Periode sind bisher nach gewiesen. Das gebört auch unter dasKapitel Wertrernichtung durch den Totenkult. ${ }^{32}$ ) Allerdings dürfen wir rechnen, daß man in den meisten Fällen nicht die besten und nevesten Falurzenge auf dem Scheiterhaufen verbrannt oder ins Grab gelegt hat. Hübṣche Einblicke in die pia fraus bei Boots- und Schiiffsbegräbnissen gewährt der Osebergfund. Das Schiff, in dem man die Vestfoldkönigin bestattete, war eine prachtroll verzierte Yacht, aber als es in die Erde kam, war es bereits längere Zeit nicht mehr seetüchtig und nur für den Grabgebranch wieder in Stand gesetzt. Ebenso waren die Pferde und Hunde, die man ihr mitgab, alte und darum זreniger wertvolle Tiere. ${ }^{33}$ )

Gegenüber den Bootsgräbern ist die Zahl der Schiffsgräber verschwindend klein, wenn man dazu alle jene Fahrzenge rechnet, die über $15 \mathrm{~m}$ lang und besonders reich ausgestattet sind. Die Hauptgruppe liegt in Vestfold, zu ihnen gehören die berühmtesten Wikingerschiffe, die von Gokstad, Tune und Oseberg, zwei kleinere Gruppen auf Rolvsöy (Smalene) und auf Karmö (nördlich von Stávanger). Dazu kommen zwei Einzelfunde in Nordfjord und in Namdalen. .

31) Upsala: E. Hildebrand, Månadsblad 1876 S. 250 ff.; K. Stjerna, Antikvarisk tỉdskrift för Sverige 18: 4, S. 49 ff.; B. Nerman, Vilka konungar ligga i Uppsala högar? 1913 ; 0. Montelius, Nordisk tidskrift 1918, S. 213 ff.; H. J u n g n e r, Fornvännen 1919 S. 79 ff.; - Byrkje: Schetelig, Vestlandske graver S. 90 ff.; Björnes, Aarsberetn, 1904 S. $200 ; 1905$ S. $353 ; 1906$ S. 341 ; - Wendel: H. Stolpe und T. J. Arne, Graffältet vid Vendel 1912.

32) Vgl. den nicht sehr ergiebigen Aufsatz von H. Schurtz, Zeitschrift für Sozialmissenschaft I (1898). Zeugnisse aus. dem germanischen Altertum, Chadwick, Cult of Odin S. 22 fi.

33) Osebergtundet I 216. 
In den norwegischen Schiffsgräbern erreicht die gegenständliche $A$ bspiegelung der Totenbootsidee ilire Akme. Mit diesen Schiffen konnten die Toten tatsächlich über das Meer fahren, alles was zur Ausrüstung des Fahrzenges gehörte, war dabei: Proviant, Kleidung, Waften, Schmuck, nichts fehlte. Und wenn am jenseitigen Ufer za Pferd, auf Wagen orler im Schlitten weitergereist werden mußte, so war auch dafür Sorge getragen.

Dann sinkt der Grabritus von der getreuen Materialisation der Idee allmählich zum symbolischen Andeuten zurück. Ein erster Schritt auf diesern Wege ist ein Bootsgrab, das im Jahre 1913 bei Kolneholme in Nerike aufgedeckt wurde. ${ }^{\dot{3} 4}$ ) Das Boot war kein brauchbares Fahrzeug, sondern eine Nachbildung. Die Steven bildeten zwei in den Erdboden eingeschlagene runde Pfähle. Der Rand bestand aus einigen Planken an jeder Seite, die lose zusammengefügt waren. Ein Boden fehlte, ihn ersetzte der flache Boden des Grabes. Darin lag der Leichenbrand und die Grabbeigaben, nach denen es in die Wikingerzeit gẹhört. ${ }^{35}$ )

Noch weiter von der Wirklichkeit entfernt sich ein Bootsgrab von Ölbör im südwestlichen Norwegen. ${ }^{36}$ ) Die Bootsform ist hier aus aufrecht gestellten, etwas nach außen geneigten Steinplatten nachgeahmt; die von einer Steinpackung umgeben unter einem Rundhügel lagẹn. An jedem Ende, Bug und Heck andeutend, standen je zwei schmälere und höhere Steinplatten. Die Länge dieses Steinbootes betrug $3,50 \mathrm{~m}$. Darin waren die Reste eines verbrannten Bootes und des mit seinen Beigaben verbrannten Toten beigesetzt.

Der nächste Schritt sind die schiffsförmigen Steinsetzungen. ${ }^{3 \top}$ ) Eine der größten und besterhaltensten ist die von Kåseberga in Schonen. ${ }^{38}$ ) Die alte Neigung des Nordens zum Steingräberbau ist hier wieder herrorgebrochen und hat ein imposantes Denkmal geschaffen. Es besteht aus 60 bis über $2 \mathrm{~m}$ hohen Steinblöcken - zwei davon sind ergänzt -, die in Zwischenräumen aneinander gereiht die spitzovale Grundform eines Schiffes nachahmen, eines sehr großen Fahrzeuges, denn die Länge des Gebildes beträgt $67 \mathrm{~m}$. Ein Stein auf der SO-Seite, vor der Spitze des Schiffes, weist nach dem Meere, an anderer befindet sich an der Heckseite. Das letzte Glied dieser Kette sind die Schiffsbilder auf den nordischen Grabsteinen. Natürlich meine ich hier keine chronologische Abfolge. Der Grabstein von Häggeby in Uppland, der als einzige Abbildung ein Schiff oline Segel trägt, das von Ruderern vorwärts getriebën wird, stammt aus der Z̈eit um 500 n. Chr. ${ }^{39}$ ) S. 48 ff.

34) 0 . Montelius, Minnesskrift 1907-1917. Utgiven af Ystads forminnesförening 1917

35) Fornvännen 1913 S. 310.

36) Stavanger Museum, Aarshefte for 1902 S. 59.

37) Montelius, Svenska fornminnes foreningens tidskrift I (1886), S. 149 ff.

38) Montelius, Ystads fornm. for. minñeskrift 1917 S. .41 ff.

39) Schweden.

Häggeby, Uppland. Schiff ohne Segel: Die Zahl der Ruder ist beinahe ebenso groß wie bei dem Boot von Nydam. Um $500 \mathrm{n}$. Chr. Arne, Srenska fornm. foren. tidskrift XI 321. 
Der Zusammenhang .dieses Steines mit den. gotländischen Grabstelen sichert die Deutung dieser Schiffsdarstellung. Auf dem vorzüglichsten der Grabsteine, dem von Stenkyrka auf Gotland (Abb. 5) ist die Scliffisfahrt ins Jenseits und das Jenseits zugleich dargestellt. Er ist in einem sanberen und technisch sicheren Reliefcreusé, deutlich nach einer gewirkten Vorlage, einem Teppich, Gobelin oder dgl., ausgeführt. Die trapezförmige, sich nach oben rerjüngende Fläche hat der Künstler in drei horizontale Streifen geteilt. Die untere füllt ein mit Kriegern besetztes, unter günstigem Winde dahin segelndes Schiff, dessen Wimpel noch in das obere Feld hineinragt. Die oberen Bildfelder zeigen den Empfang und die Bewillkommnung in Walhall. Die Idee von der Bootsfahrt ins Jenseits läß̣t sich also bei den nordgermanischen Stämmen im Totenkult zuerst von $300-500$ in der Grabausstener, dann ron 500 bis 1000 und später im Grabbau und in der., Grabausschmückung verfolgen.

Wie steht es nun um den Ursprung dieser Idee? Die Zeit und die Zusammenhänge, in der sie auftritt, und die äußere Form, in der sie zuerst archäologisch greifbar wird, nämlich die Mitgabe von Totenpfennigen für

$\therefore$ den Fährmaun, legen es nahe, nach den Mittelmeerländern zu blicken. In der Tat ist diese Aniffassung mehrfach, besonders ron Stjeria vertreten morden. ${ }^{+0}$ )

Tulstörp, Vermenhögs hd., Schonen. Drachenschiff Bug und Heck laufen in Tierköpfe aus. Längs der Relinge 14 Schilde. Über dem Schiff ein phantastisches Tier, wie auf den orientalischen Silberschalen: Runenstein des dänischen Sprachgebietes. Ende i.es 10. Jahrhunderts. L. Wimmer, Danske runemindesmæerker IV 26. Handausgabe 1914 No. 22.

\section{Gotland.}

Bro. Schiff mit Ruderbänken, einer Art von Kajüte. Nordin in Montelius-Festschrift 1903 S. 145 Abb. 3.

Stenbro. Rudimentäre Schiffisdarstellung. Nordin S. 147.

Högbro. Schiff mit Mast; Segeln und Schilden, ohne Reiter. Nordin Abb. 5.

Tjängvide. Schiff bemannt mit Mast und Segeln. Oben ein bewaffineter Reiter auf einem achtfüßigen Roß (Odin?) und eine Frauenfigur. Montelius, Kulturgeschichte Schwedens 1906 S. 135 Abb. 24; S. Bugge, Antikv. tidskrift 5, S. 103.

Levede. Fahrt auf einem Wagen, roran läuft ein Hund. Runen. Studier tillägnadeHenrik Schück 1905, S. 123 .Abb: 3.

Stenkyrka. Schönster erhaltener Bildstein. (Abb. 5.) Unten ein mit Kriegern bemanntes Schiff, oben Walhall. Nordin S. 149.

Norwegen.

Austreim Nordfjord. Einfache Schiffsdarstellung. Wahrscheinlich ist dieser Bildstein auf Gotland zurückzuführen. A. Bugge, Norges Historie I 119.

bänemark.

Spentrup, Amt Randers. Schiff mit Mast. Runenstein, iragmentiert. Ende des 10. Jahrbunderts. Wimmer II 21, Handausgabe No. 21.

Hjærmind, Amt Viborg, Lmriß eines Wikingerschiffes. Ende des 10. Jahrhunderts. Wimmer No. 24.

Sönder Kirkeby, Amt Maribo. Oben ein Schiff. Ende des 10. Jahrhunderts. Wimmer II 438.

40) K. Stjerna, Essays on Beowulf 1912 S. $97 \mathrm{ff}$. 


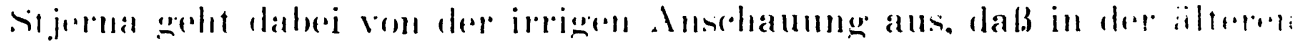

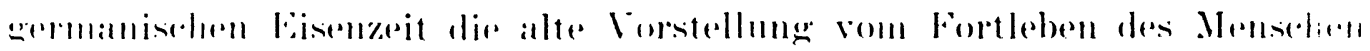

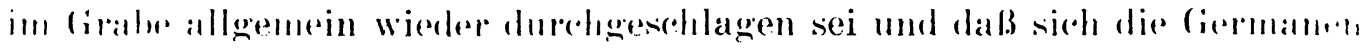

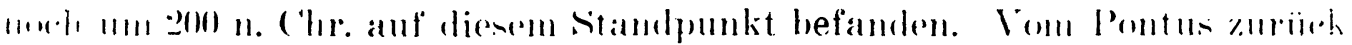

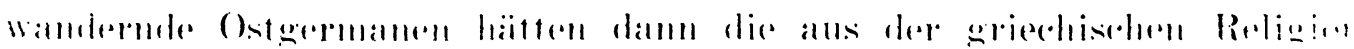

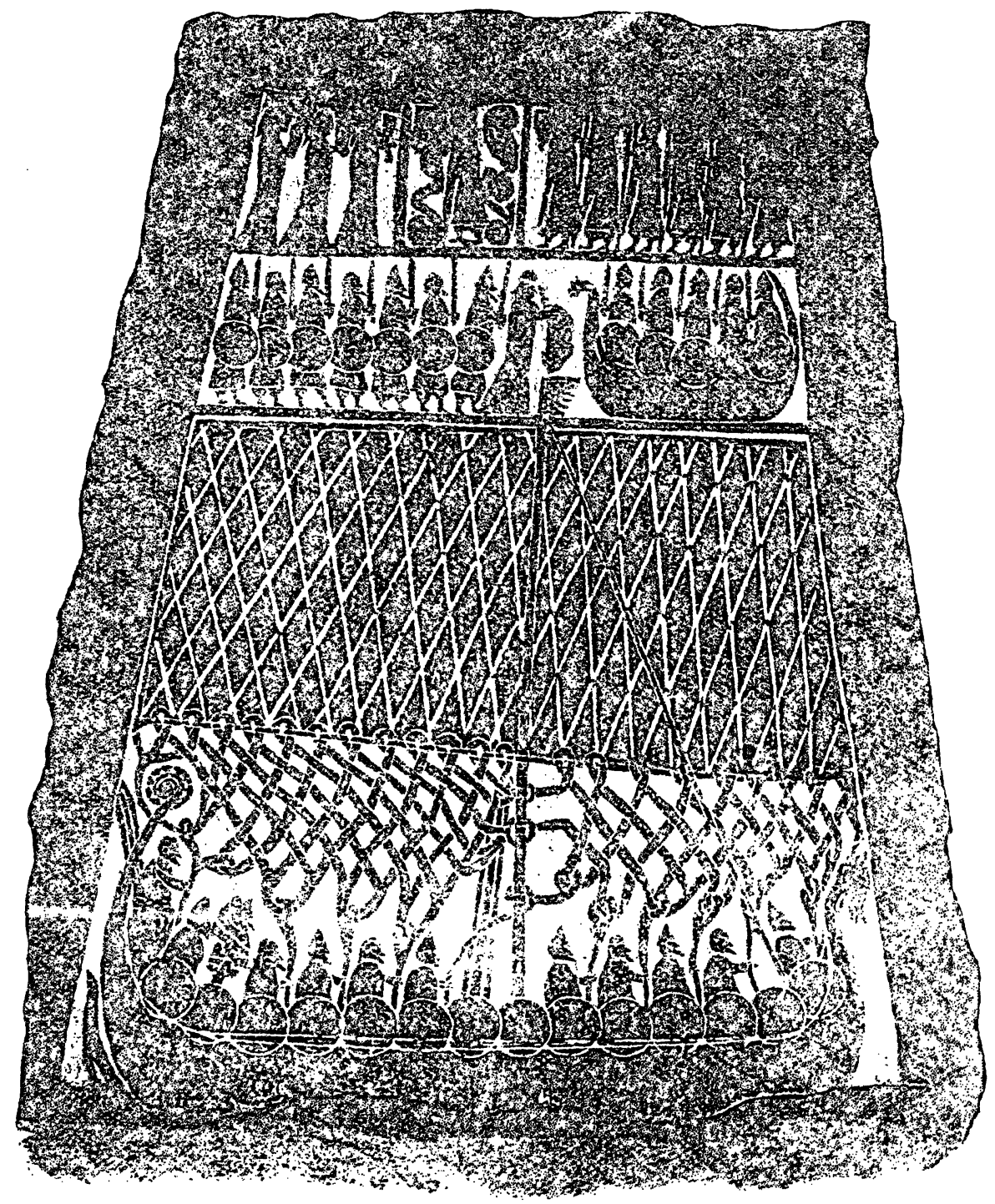

.1bis. $\therefore$

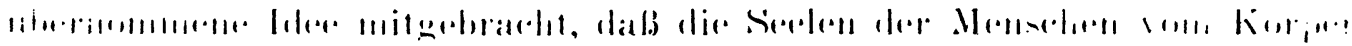

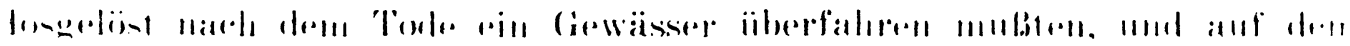

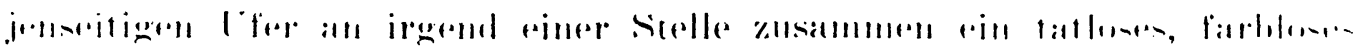
liar.in liilurtenl.

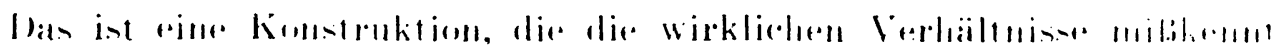

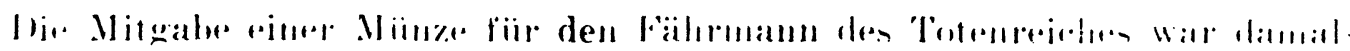

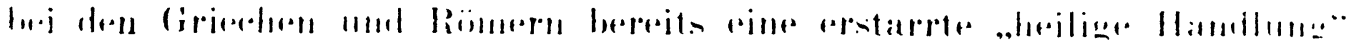


„Daß es Manen gibt und ein unterirdisches Reich, einen Fährmann mit seiner Stange und schwarze Frösche im stygischen Schlunde, daß so viele Tausend Menschen in einem einzigen winzigen Nachen über das Wassersetzen können, das glauben nicht einmal mehr die Kinder.“" ${ }^{+1}$ ) Der alte griẹchisch-römische Glaube fristete seinẹ Kult in krausen Zeremonien und Aberglauben. Seine Götter und Heroen führten ein rein literarisches Leben. Auf seinem eigenen Gebiet unterlag er den fremdländischen, insbesondere den orientalischen Religionen. Schwer zu glauben, daß gerade von ihm ganz neue Vorstellungen vom Leben nach dem Tode ạusgegangen seien.

Über die Jenseitsvorstellungen, die damals die Germanen am Pontus vorfanden, sind wir zunächst durch die Grabstelen und die in Rostowzents Werken zusammenfassend behandelten Grabanlagen der römischen Zeit in Pantikapaion ${ }^{43}$ ) einigermaßen unterrichtet. Auf den Kertscher Katakombengemälden ist nichts zu verspüren von dem tristen homerischen Totenglauben oder der welmmütigen Resignation der attischen Grabreliefs und weißgrundigen Lekpthen. Da sieht man auf der einen Seite, daß der im Mutterlande blühende Mysterienkult auch hier Wurzeln gescllagen hat, und auf der andern Seite zeigen große Bilder mit Abschiedsmählern, Szenen aus dem Landleben, Kampfdarstellungen u. a., daß das diesseitige Leben mit sehr realen Zügen in das Reich der Toten hineintritt. Die robusten Landjunker auf der Krim hatten handgreifliche und persönliche Vorstellnngen von dem jenseitigen Lebęn, das ihnen in Aussicht gestellt war. ${ }^{44}$ )

Auch asiatische Kultẹ werdèn in dieser: zweiten Blüteperiode des Bosporusgebietes - worüber wir im einzelnen nicht viel wissen - hei der zunehmenden Barbarisierung Einlaß gefunden haben. Denn der östlicheTeil, das bosporanische Reich, unterliegt in dieser Spätzeit immer imehr aus Asien kommen Einwirkungen. Ferner, in Pantikapaion, Theodosia, Gorgippia, Phanagoria, Tanaïs gibt es damals religiöse Thiasen, die aus Kleinasien stanunten. Die Religion dieser „Brüder“, zum großen Teil gräcisierte Barbaren, war eine Mischung von Judentum und Heidentum. Sie verehrten den sehr hohen, den höchsten Gott, den ewigen Gott, hatten aus der giriechischen

41) Juvenal II 149.

42) v. Kieseritzky und Watzinger, Griechische Grabreliefs aus Südrußland 1909.

43) M. Rostowzew; Die antike dekorative Malerei in Südrußland 〈russisch〉. St. Petersburg 1914. Band I (Text), Band II (Atlas).

44) Ich habe schon früher (Praeh. Zeitschi. I 69) auf die.merkwürdigen Zeichen hingewiesen, die auf den Wänden der Kertscher Katakomben, wie auch sonst vielfach in Südrufland vorkommen. Ich habe sie ins 1.-3. Jahrhundert datiert. Rostowzew stimmt diesem Ansatz zu. Es ist für die Entstehung und Ausbreitungsfrage der ältesten Runen wichtig, daß sie auch auf den Runenlanzenspitzen von Brest-Litowsk und Mlüncheberg (vgl. Götze, Mannus I) und auf Stücken der nordischen Moorfunde auftreten. Es sei noch auf die Arbeit von Ramsay, Journal of hellenic studies 32 (1912) S. 151 ff. hin!gewiesen, der die Inschriften phrygischer Mysten studiert. Sie waren durch die Kenntnis gewisser geheimer Zeichen (тér $\mu \omega g$ ) verbunden und feierten gemeinsame heilige

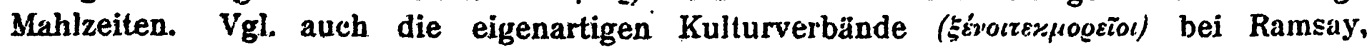
Studies in the history and art of the Eastern provinces of the Roman empire 1906. 
Religion die Zeusvorstellung beibehalten nnd lehnten viele Vorschriften der Synagoge ab. ${ }^{45}$ ) Eudlich darf man die.mancherlei Kulte nicht vergessen, die das römische Heer mit sich brachte. Olbia und Chersonesos waren längere Zeit Standquartiere römischer Legionen. ${ }^{40}$ ) In Ai-Torlor ist ein Legionslager aufgedeckt, ein Tempel mit Votivgaben und Reste eines Mithräums. ${ }^{ \pm 7}$ )

Das 3. Jahrhundert, in dem die Goten sich in Südrußland festsetzten und ihre Macht nach allen Seiten auslehnten, ist zugleich auch die Zeit, in welcher die iranischen Mysterien den größten Einfluß in der römischen Welt gewannen und zu einer Art von Staatsreligion wurden. ${ }^{48}$ )

Sehr stark war ihre Verbreitung damals wie schon frïher in den benachbarten römischen Balkanprovinzen, Moesien und Dacien. In Carnuntum weihten im Jahre 307. Diokletian, Galerius ind Licinius den Erweite'rungsbau eines Mithräums „fautori imperii sui“.49) Was den Mithracismus wịe den anderen Mysterien ihren Einfluß auf die breiten Massen nicht uur, sondern auch auf die Elite der Gesellschaft schuf, war, daß sie ihren Anhängern lichtere Ausblicke in das jenseitige Leben gab, als der alte griechisch-römische Pa'ganismus. Der Mensch lebt nicht jenseits ron Styx und Acheron als schwacher, tatenloser Schatten oder zarter Hauch fort, sondern im Vollbesitz seines Leibes und seiner Seele. So lauteten jedenfalls die Lehren der Mysterien, die in der griechisch-römischen. Welt gefeiert wurden. $\left.{ }^{50}\right)^{\cdot}$. Die schwankendẹn und trostlosen Vorstellungen, die clas antike Heidentum hegte, verwandelten sich in die verbürgte Hoffnung auf eine bestimmte Form der Seligkeit.

Diese Kulte und Mysterien sind es also, die damals auch an den südöstlichen Grenzen des Imperium Romanum das religiöse Denken der Menschen beherrschte. Mit ihnen, nicht mit dem nur noch in der Literatur und entlegenen Dörfern Griechenlands und Italiens fortlebenden Paganismus mußten also die Germanen zusammentreffen.

Der alte homerisclie Totenglaube von Charon, Styx und Hades hat.

45) E. von Stern, Theodosia und seine Keramik S. 21 ff.; E. Schürer in SB Berlin 1897 S. $201 \mathrm{ffi}$; F. Cumont, Hypsistos im Supplementband der Revue de l'Instruction publique en Belgique 1897.

46) v. Friesen, Om runskriftens härkomst $1904 \mathrm{~S}$. $45 \mathrm{ff}$. gibt sich große Nühe zur Erklärung des lateịnischen Einschlages im germanischen Runenalphabet nachzuweisen, daß ein lateinisches Kulturzentrum sich in der Nachbarschaft der Goten an der untersten Donau befunden. Das ist nicht nötig. Wir besitzen aus Südrußland selbst genug Material, daß für einen in diesem Belang hinreịchen großen römischen Einfluß spricht. Als eines der spätesten Denkmäler noch ein zweisprachiges Dekret des Septinius Severus, das den Bürgern von Tyras Abgabefreiheit für Ein- und Ausfụhr geivährt. Latyshev, Inscriptiones antiquae orae septentrionalis Ponti Euxini 1 No. 3.

47) Rostowzew, Das Heiligtum der thräkischen Götter und die Inschriften der beneficarii in Aï-Todor. Iswestja der archäol. Kommission 40.(1911) S. $1 \mathrm{ff}$.

48). F. Cumont, Die Mysterien des Mithra 19112 S. 78 ff.

49) F. Cumont, Textes et monuments figurés relatifs aux mystères de Mithra 1899 II S: 491 No. 227.

50), Reitzenstein, Archiv f. Religionswissenschaft 6 (1904) S. $406 \mathrm{ff}$. 
mit der germanischen Jenseitsvorstellung nichts zu tun. Im freien Germanien, müssen wir vielmehr sagen, bilden sich in der römischen Kaiserzeî Vorstellungen über die Bootsfahrt ins Jenseits, die so bestimmt geformt sind, daß sie ihren Ausdruck im Grabritus finden. Im Halbdnukel des religiösen Bewußtseins haben sie wahrscheinlich vielerorts gelebt. Die Sitte des Totenpfennigs kann nichts anderes sein als die äußerliche Übernalıme eines antiken Grabbrauchs, hinter dem kein tieferer Sinn melhr liegt. Die damals im Römerreich rirklich iebendigen Gedanken über das jenseitige Leben haben eine ganz andere Wirkung auf die Anschaunngen der Germanen ausgeübt. Darauf kann ich aber hier nicht eingehen.

Ich war zu dieser Ausicht über die Sache bereits im Jahre 1915 gekommen und hatte sie im Soimmer 1918 bestimmt formuliert, ohne Kenntnis der in dieser Zeit in Schweden erschienenen Literatur und neuerer Ausgrabungsergebnisse. Diese zeigen num in der Tat, daß die Torstellung von der Bootsfahrt ịs Jenseits, wie auch bei ihrer weiten Verbreitung zu erwarten war, bereits sehr viel früher im Norden auftauchte und damn viele Jahrhunderte lang röllig verschwunden scheint, bis sie in der römischen Kaiserzeit zu neuem Leben erwacht. Das darf nicht überraschen. In dem Komplex der Vorstellnngeu, die sich um Grab und Jenseits berregen, gibt es keine Art ron logischer Abfolge oder generatio spontanea als bestimmendes Prinzip. Der historische Durchschnitt durch die sulizessiven Schichten der Riten des Totenkultus und der Torstellungen, die ihnen zugrunde liegen, zeigt überall Ubberschneidungen und Widersprüche, Uraltes neben ganz Jungem. Die religiồșen Ideen haben eine wunderbare Lebenskraft. Sie entstehen, dauein und sinken dann in die Tiefe, aber da unten halten sie sich mit unausrottbarer Zähịkeit, und eines Tages erscheinen sie wieder auf der Oberfläche.

Am Ende der jüngeren Steinzeit bestattete man den Toten im Norden hekanntlich in unter die Erde gelegten Kisten aus behauenen oder gesl:altenen Steinplatten. Die Sitte nält sich in Skandinavien bis in die ältere Bronzezeit, in Meklenburg haben wir keine Belege dafür,,51) in den dentschen südlichen und westlichen Nachbarländern taucht sie in der II: Periode wieder auf. Die Steinkiste ist eine verkleinerte Kammer, ursprünglich für die Massenbestattung bestimı̨nt, erst allmählich zum Einzelgrab gerworden. Der Sarg tritt zum ersten Mal in der älteren Bronzezeit auf, in der Form der Eichbaumsärge: ein runder 'Stamm, der in der Längsrichtung in zrei gleiche Teile gespalten und ausgehöhlt ist. Die eine Hälfte diente als Lager, die andere als Deckel. ${ }^{52}$ ) Der Sarg tritt also verhältnismäßig spät auf, immerhin noch erheblich früher wie z. B. in Griechenland. Dort bestattet man den Toten in mykenịscher Zeit olıne Sarg, wenn die spätmykenischen

51) R. Beltz, Montelius-Festschrift 1913 S. 91.

52) Montelius, Svenska fornm. foren. tidskuift 9 (1896) S. $77 \mathrm{ff}$; ders., Die vorclassische Chronologie Italiens 1912 S. 48f.; Eichbaumsärge schon aus der jüngeren Steinzeit in Schleswig-Holstein: Splieth 40, Bericht des Schleswig-Holsteinischen Museuns vaterländischer Alterlimer 1894 S. 17, 19. 
Tonkisten wirklich nur lokale Erscheinungen sind.53) Auch in der Dipylonzeit fehlen sie noch. Erst die Brennsitte scheint auf dem Weg über die Aschenurne zur Einführung des Sarges geführt zu haben. Die Verwendung der germanischen Baumsärge hängt nicht mit dem Aufkommen des Leichenbrandes zusammen. Es läßt sich vielmehr beobachten, wie die Form erst allmählich dem neuen Ritus angepaßt wird. Es muß zugegeben werden, daß die Einführung des Holzsarges eine bedeutungsvolle Neuerung in Grabbrauch ist. Ungefähr gleichzeitig damit treten im südlichen Skandinavien die viel behandelten Felszeichnungen (,hällristningar") auf, ${ }^{54}$ ) und man ist neuerdings geneigt, beides in einen Zusammenhang zu bringen. ${ }^{55}$ ) Bei den Deutungsversuchen an den „Hällristningar“ ist man zu drei verschiedenen Resultaten gekommen. Die einen sehen in ihnen reine Produkte einer unbeholfenen, spielerischen Bildkunst, die anderen Erzählungen historischer Art, in denen die Skandinavier der Bronzezeit Begebnisse der Nachwelt überliefern wollten, die dritten endlich Darstellungen mit magischreligiösem Sinn. Die letztere Ansicht wird besonders von Almgren 56) und Ekholm vertreten und erhält in der Tat dadurch eine kräftige Stütze, daß ähnliche Darstellungen auch auf den Platten von Steinkistengräbern rorkommen und hier mit dem Totenkultus in Verbindung gebracht werden müssen. Am bekanntesten sind die oft wiedergegebenen Bilder auf der Innenseite von Platten, die zu einer im Jahre 1750 bei Kivik (Schonen) aufgedeckten Steinkiste gehören. ${ }^{57}$ ) Vereinzelt kommen diese Grabdarstellungen in Dänemark (Seeland) und Norwegen (Rege); häufiger in Schweden, Hessen, Ostthüringen und Hannover vor. ${ }^{58}$ )

Sehr beliebte Motive auf diesen Steinkistenplatten sind Schiffe, Fußsohlen und Schälchen. Ekholm meint nun, da die Schälchen und Fußsohlen einen deutlichen Bezug auf den Totenglauben hätten, so müsse dasselbe auch für die Schiffe geschlossen werden; und sieht in eben diesen Schiffsdarstellungen den Beweis dafür, daß man bereits in der älteren Bronzezeit an eine Bootsfahrt ins Jenseits glaubte, ganz ähnlich wie in der. Völkerwanderungs- und Wikingerperiode.

Dà dieselben Bilder, namentlich die Schiffe, auch auf den Fielszeichnungen erscheinen, die „Hällristningar“ aber in einem Zeitpunkt auftreten, wo die Steinkisten verschwinden und die Sargbestattung: Sitte wird, so hält er dafür, daß : auch die „Hällristningar“ ebenfalls mit dem Totenkult zu-

53) Dragendorff, Thera II 90 ; vgl. Mariani, Monumenti dei Lincei VI $34 \mathrm{ff}$.

54) Vgl. die Literaturnachweise bei Montelius, Kulturgeschichte Schwedens. 1906 S. 126.

55) G. Ekholm, De skandinaviska hällristningar oc̣ deras betydelse. Ymer 1916; ders., Upplands fornminnes fören. tidskrift VII S. 175 ff.; A. Nordén, Hällisistningars kronologi och betydelse. Ymer 1917.

56) O. Almgren, Bidrag till Göteborgs och Bohusläns fornm. och historia VIII (1906-1913) S. 570.

57) 0. Montelius, Svenska fornm. fören. tidskrift 10 (1900) S. 193 ff.

58) Kossinna, Korrespondenzblatt des Gesamtvereins 1908, S. . 343 ff. Schumacher, VIII. Bericht der Römisch-Germanischen Kommisssion 1917 S. 54. 
sammenhängen und ihre Bilder so erklärt werden müssen. Alle die zahlreichen Boote und Schiffe auf den skandinavischen Bergtafeln wären also Darstellungen von Totenschiffen und von der Fahrt. ins Jenseits.

Die Kombinationen Ekholms stehen auf einer schmalen Basis und bedürfen, um wirkliches Gewicht zu erhalten, weiterer Stützen. Diese sind nun in der Tat in den letzten Jahren durch neue Beobachtungen auf der Insel Gotland beigebracht. Die Resultate der von Schnittger und H. Hansson durchgeführten Grabungen sind noch nicht veröffentlicht. Ich möchte nicht vorgreifen. Freundlichen Mitteilungen von O. Almgren und B. Schnittger entnehme ich hier Folgendes: Auf Gotland finden. sich eine große Zahl schiffsförmiger Steinsetzungen. : Die untersuchten Anlagen gehören ent-

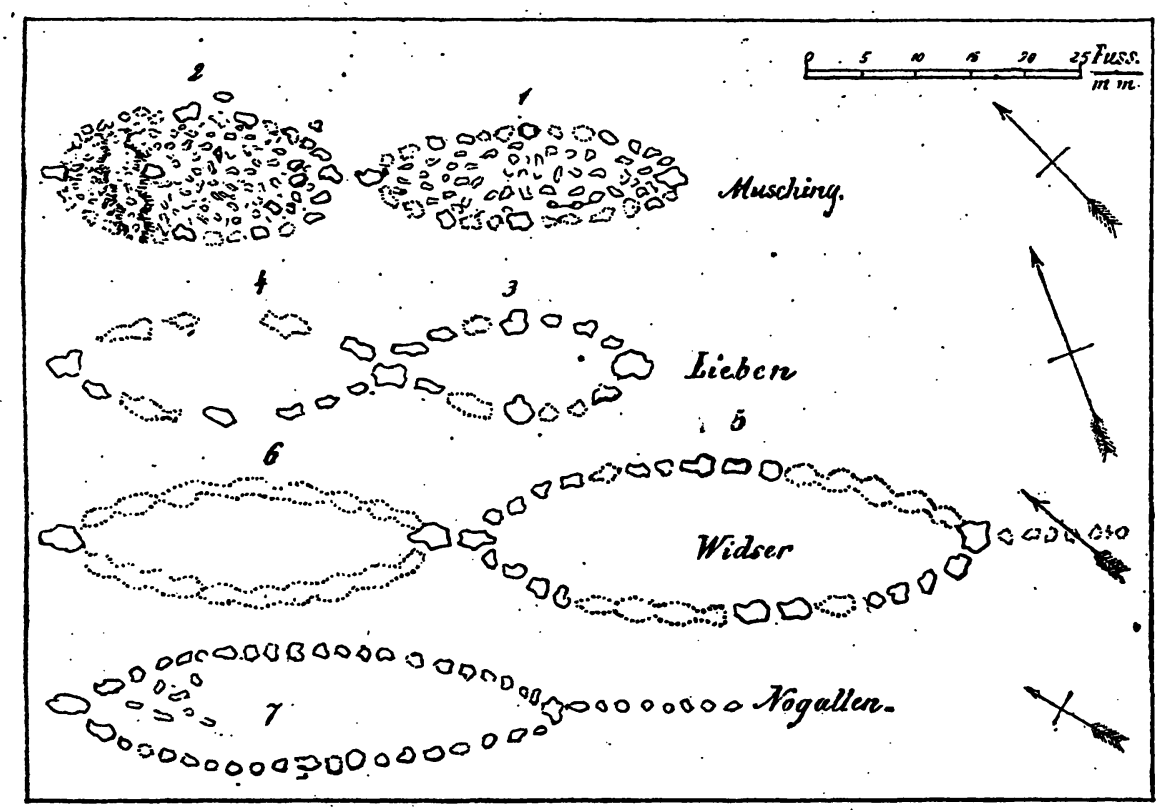

Abb. 6.

weder der jüngeren Bronzezeit (Leichenbrand in Urnen) oder der İ. Periode der älteren Eisenzeit (Skelette in nachlässig gebauten Steinkisten unter der Erdoberfläche) an. Das läßt nun allerdings keinem Zweifel mehr Raum, daß die Vorstellung von der Bootsfahrt ins Jenseits im Norden bereits während der Bronzezeit geherrscht und sich bis ungefähr $300 \mathrm{v}$. Chr. wenn neue Grabungen dieses Datum nicht noch weiter heruntersetzen gehalten hat. Vermutlich ist die Idee schon in der älteren Bronzezeit entstanden, und die Bestattung in Eichensärgen und die Schiffsbilder auf den Grabplatten und Felszeichnungen stehen damit im Zusammenhang.

Damit rücken nun auch die von Grewingk publizierten kurländischen Schiffsgräber am Rigaischen Meerbusen aus ihrer Vereinsamung in einen festen Zusammenhang. ${ }^{59}$ ) Auch sie bestehen aus schiffsförmigen Steinsetzungen (meist paarweise hintereinander liegend) und enthalten in kleinen Steinkisten Urnen mit Leichenbrand (Abb. 6). Von Metallgegenständen

59) C. Grewingk, Die Steinschiffe von Musching. Dorpat 1878. 
enthielt die Steinkiste des einen Schiffes eine „aus Erz, Bronze oder Messing bestehende . . . fingerlange Dolchklinge“. Die Stellung dieser Gräber innerhalb der baltischen Entwicklung war bisher ziemlich zweifelhaft. Gegenstücke dazu in Kurland, Livland und Estland gab es nicht, denn dạs „Steinschiff“ von Slahweek und die übrigen baltischen Steinreihengräber haben damit nichts zu tun. ${ }^{60}$ ) Jetzt werden wir die Schiffssetzungen des. Talsener Kreises mit Sicherheit in die Bronzezeit datieren und im Zusammenhang mit den gotländischen Gräbern auf skandinavische Kolonisation der kurländischen Küste während dieser Zeit schließen könnèn.

Wir haben also zwei Perioden, in denen die Idee von einer Bootsfahrt ins Jenseits den germanischen Totenkultus beeinflußt. Die erste umfaßt die.Bronzezeit und die I. Periode der älteren Eisenzeit. Zur vollkommenen Materialisation der Idee, zur Bestattung in Booten und Schiffen kommt es in diesen zwei Perioden nicht. ${ }^{61}$ ) Es bleibt bei Substitutionen: das Wirkliche wird durch ein Bild oder durch eine andeutende Wiedergabe in anderem, nicht geeignetem Material ersetzt. ${ }^{62}$ )

Sieben bis acht Jahrhunderte später erscheint die Vorstellung im Grabritus der Nordgermanen von neuem: Diesmal kleidet sie sich anfangs in das Gewand antiken Brauches. Um 500 hat man bereits die letzten Konsequenzen in der Richtung auf eine vollständige Realisation der Vorstellung gezogen und bestattet die Toten in einem wirklichen Boot. Einige Jahrhunderte später ist die Sitte in großen Teilen Skandinaviens stark verbreitet und erreicht ihren Gipfelpunkt in den Schiffsbegräbnissen norwegischer Könige. Die Substitution durch Bilder (Grabsteine) tritt früh ebenfalls schon um $500 \mathrm{n}$. Chr. - auf und es bleibt dahingestellt, ob sie zu den Anfangs- oder Verfallserscheinungen (pia fraus) im Grabritus zu rechnen sind. Das Absterben der Sitte zeigen die aus Holz und Stein konstruierten Boote und die schiffsförmigen Steinsetzungen. Möglicherweise spüren wir darin schon den Einfluß der neuen christlichen Lehre.

60) Doch vgl. Praehist. Zeitschrift 5 (1913). S. 52.

61) Bei der Beschreibung des Grabiundes von Vaale (Mitteilungen des Anthrop. Vereins in Schleswig-Holstein 14 (1901) S. 13) ist von den Überresten eines Bootes, das als Sarg benutzt war, die Rede (II. Periode der Bronzezeit). Nach freundlicher Mitteilung F. Knorrs handelt es sich um einen trogartig ausgehöhlten Eichenstamm der gewöhnlichen Art.

62) Wahrscheinlich gehören hierzu auch die kleinen goldenen Boote im Funde von Nors (Jütland). S. Müller, Nordische Altertumskunde I 422: 